\title{
Rotura ventricular após substituição da valva mitral
}

\author{
Marcelo Campos CHRISTO*, Liberato S. Siqueira de SOUZA**, Marcilio José STORTINI**, Sérgio L. da \\ Costa TEIXEIRA**, Osvaldo CRUZ JÚNIOR**
}

RBCCV 44205-79

\begin{abstract}
CHRISTO, M. C.; SOUZA, L. S. S.; STORTINI, M. J.; TEIXEIRA, S. L. C.; CRUZ JÚNIOR, O. - Rotura ventricular após substituiçăo da valva mitral. Rev. Bras. Cir. Cardiovasc., 4(1): 75-83, 1989.
\end{abstract}

RESUMO: Entre 1979 e 1988, 4 casos de rotura do ventrículo esquerdo (VE), após substituição da valva mitral (SVM), foram registrados entre 332 pacientes. Os autores reconhecem, entre seus casos, 3 roturas na junção atrioventricular, ocorridas logo após a saída de by-pass e 1 rotura em parede posterior do VE, ocorrida na unidade de terapia intensiva (UTI). Os pacientes eram todos do sexo feminino e tinham, em média, 58 anos de idade. Tais acidentes aconteceram 3 vezes após substituição isolada da valva mitral e 1 vez em operação combinada de SVM e revascularizaçăo do miocárdio. Admitem que o mecanismo principal da rotura está ligado à criação de zona de acinesia isquêmica, localizada em parede do VE, secundária à superdistensão de anel mitral. Relacionam o desencadeamento dos acidentes com a superdistensāo dos anéis valvares, avaliados com medidores inadequados, usados em coraçóes profundamente relaxados pela cardioplegia. Preconizam modificaçōes na cabeça desses medidores, adaptando-os a cabos maleáveis, de modo a permitir um posicionamento mais perfeito da cabeça do medidor do anel mitral. Admitem que o perfil mais ou menos elevado das próteses não parece haver influenciado no aparecimento, ou na prevenção desses acidentes, mas sim na determinação do tipo anatómico da lesão. Estão de acordo com outros autores, quando admitem que a fragilidade do miocárdio, em pacientes idosos, agravada com a remoção do aparelho valvar mitral (ventrículo sem sustentação), criaria condiçóes para o aparecimento desses acidentes. Consideram desejável a preservação do aparelho valvar mitral nas SVM, mas consideram que técnicas seguras, com essa finalidade, precisam ser ainda desenvolvidas.

DESCRITORES: rotura ventricular, cirurgia; valvas cardiacas, cirurgia.

\section{INTRODUÇÃO}

Em 1967, ROBERTS \& MORROW ${ }^{24}$ descreveram, em necropsias, roturas pós-operatórias localizadas na parede póstero-lateral do ventrículo esquerdo (VE), em 2 pacientes que haviam sido submetidos a substituição da valva mitral. Em 1 deles, uma separação entre átrio esquerdo e o VE foi atribuída à remoção excessiva de grandes depósitos de cálcio que se extendiam através da cúspide mitral, atingindo o ane!, no miocárdio ventricular e, no outro, uma perfuração em parede livre do VE foi atribuída à excisão excessiva do músculo papilar.
Posteriormente, essas lesōes foram classificadas, por BJÖRK et alii ${ }^{3}$, em tipos I e II, respectivamente.

Em 1982, ROBERTS et alii ${ }^{23}$ descreveram um terceiro tipo de laceração em VE, conseqüente à incisão acidental da parede ventricular no momento da excisão instrumental da valva mitral (Figura 1).

Desde a primeira descrição, aproximadamente 100 casos foram relatados na literatura' ${ }^{1}, 3,4,8 \cdot 10,12,13,15$, $17,21,23,24,28-29$, com mortalidade uniformemente elevada, atingindo de $0,5 \%$ a $2 \%$ dos pacientes submetidos a substituição da valva mitral ${ }^{3}$. Não obstante ser, hoje,

Trabalho realizado no Hospital Felicio Rocho. Belo Horizonte, MG, Brasil.

Apresentado ao 16: Congresso Nacional de Cirurgia Cardiaca. São Paulo, SP, 7 e 8 de abril, 1989.

* Do Hospital Felicio Rocho e da Faculdade de Medicina da Universidade Federal de Minas Gerais.

** Do Hospital Felicio Rocho.

Endereço para separatas: Marcelo Campos Christo. Rua Dias Toledo, 76. Vila Paris. 30350 Belo Horizonte, MG, Brasil 


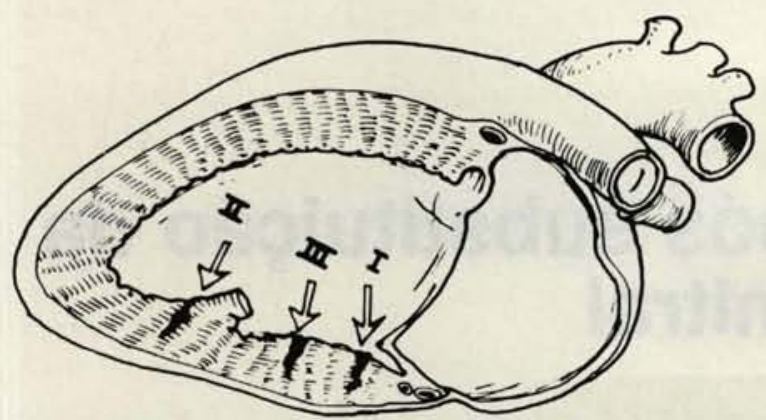

Fig. 1 - Reproduçăo esquemática de corte transversal do coraçăo esquerdo em que a valva mitral e o músculo papilar posterior foram removidos. As setas indicam a localização das roturas tipos I, II e III.

uma complicação bem reconhecida, persiste uma enorme divergênciada opiniâo sobre os mecanismos que conduzem ao seu aparecimento.

Neste trabalho: 1) estudamos 4 casos de rotura do VE após substituição da valva mitral, registrados em nosso Serviço; 2) formulamos um novo conceito sobre os mecanismos dessas roturas; 3 ) propomos cuidados técnicos básicos, destinados a prevenir essas complicaçōes.

\section{CASUISTICA E MÉTODOS}

Entre 1979 e 1988, em nosso Serviço, 332 pacientes foram submetidos a substituição da valva mitral, em operações isoladas ou combinadas.

Destes pacientes, $193(58 \%)$ receberam próteses de porcino modelo Carpentier, enquanto $135(42 \%)$ receberam próteses de porcino modelo Labcór. Quanto ao sexo: $195(58 \%)$ eram do sexo feminino e $137(42 \%)$ eram homens. Foram registrados 26 b́bitos hospitalares $(7,8 \%)$, sendo que, destes, $4(1,2 \%)$ se deveram à rotura do VE, o que constituiu a causa-mortis em $2 \%$ dos pacientes do sexo feminino e 1,2\% da mortalidade global. A idade dos nossos 332 pacientes variou de 20 a 70 anos, sendo que $60 \%$ deles (199) tinham menos de 40 anos de idade.

Em todas as nossas operaçōes, foi empregada a cardioplegia com potássio e clampeamento aórtico, com tempo médio de 80 minutos e hipotermia moderada $\left(28^{\prime} \mathrm{C}\right)$. Em 3 dos nossos casos, a rotura (rotura tipo I) ocorreu na sala de operação. Todos os 3 foram imediatamente reoperados, sem sucesso. No último paciente, a rotura ocorreu no centro de tratamento intensivo (CTI), 2 horas após a cirurgia (rotura tipo II). O paciente foi reexplorado no próprio CTI, e também foi a óbito. Nos três primeiros pacientes, a rotura era claramente observada no sulco atrioventricular, enquanto que, no último paciente, ela foi descrita na parede ventricular, entre a base do músculo papilar e sulco atrioventicular. Em todos os 4 casos, a haste mais posterior e a porção correspondente do anel da prótese pareciam empurrar para fora a parede do VE. Nos pacientes reoperados logo após a rotura, as próteses foram removidas e foi tentada a reparação da lesão.

Nessa ocasião, foi verificado que as lesōes, internamente, eram mais extensas do que apareciam externamente, orientadas no sentido da trabeculação do VE e de avaliação precisa muito difícil, por estarem sempre envolvidas por hematomas extensos.

Cumpre assinalar que, embora tivéssemos encontrado graus variáveis de calcificação nas valvas, em nenhum dos nossos casos a calcificação foi de grau muito intenso e sempre sem envolvimento importante do anel valvar. Os dados dos nossos pacientes são sumarizados na Tabela 1.

\section{RESULTADOS}

$\mathrm{Na}$ análise da nossa casuística, alguns aspectos devem ser considerados:

\section{Idade dos pacientes}

A rotura do VE após substituiçāo da valva mitral atingiu um grupo de pacientes que se situavam entre 42 e 70 anos de idade (média de 58 anos), constituindo $3 \%$ dos óbitos desse grupo. Nas séries apresentadas por COBBS Jr. et alii ${ }^{8}$, ZACHARIAS et alii ${ }^{29}$, ROBERTS \& MORROW ${ }^{24}$, BJÖRK et alii ${ }^{3}$ e DARK \& BAIN ${ }^{10}$, totalizando 44 pacientes, a média de idade foi, também, de 58 anos. Quer-nos parecer, fora de dúvida, que os pacientes mais idosos constituem um grupo de risco mais definido para este tipo de complicação.

TABELA 1

PACIENTES COM ROTURA DO VE APÓS SUBSTITUIÇÃO DA VALVA MITRAL

\begin{tabular}{lccccccc}
\hline LESÄO & DATA & IDADE & SEXO & PROTESE/CALIBRE & LESÃO & OPERAÇÃOASSOCIADA \\
\hline Tipo I & 08.03 .79 & 42 & F & Carpentier 31 & IM & Nenhuma \\
Tipo I & 15.10 .80 & 52 & F & Carpentier 33 & DLM & Nenhuma \\
Tipo I & 15.06 .82 & 70 & F & Carpentier 29 & IM I Nenhuma & Nontes de Safena \\
Tipo II & 12.02 .88 & 70 & F & Labcór 31 & IM + ICO & Pontes de \\
\hline
\end{tabular}

IM: insuficiência mitral; DLM: dupla lesāo mitral; ICO: insuficiência coronária. 
CHRISTO, M. C.; SOUZA, L. S. S.; STORTINI, M. J.; TEIXEIRA, S. L. C.; CRUZ JÚNIOR, O. - Rotura ventricular após substituiçăo da valva mitral. Rev. Bras. Cir. Cardiovasc., 4(1): 75-83, 1989.

\section{Sexo}

As roturas de VE atingiram $2 \%$ dos nossos 195 pacientes do sexo feminino, que contituíram $58 \%$ dos nossos 332 pacientes. Nenhum acidente semelhante ocorreu entre os 135 pacientes masculinos operados. Nossos dados coincidem com os colhidos na literatura: de 67 casos de BJÖRK et alii ${ }^{3}$, KATSBIE et alii ${ }^{17}$ COOBS Jr. et alii ${ }^{8}$, SPENCER et alii ${ }^{26}$, GOSALBEZ et alii ${ }^{15}$, TREASURE et alii ${ }^{27}$, ZACHARIAS et alii ${ }^{29}$ e DARK \& BAIN ${ }^{10}, 85 \%$ eram do sexo feminino.

\section{Próteses}

As próteses de pocino começaram a ser empregadas a partir de abril de 1978 e a primeira rotura de VE foi reconhecida em maio de 1979 . Em todos os nossos casos, a haste da prótese, mais posteriormente situada, e a porção correspondente do anel pareciam fazer saliência na lesão, na junção atrioventricular e/ou na parede livre posterolateral de VE; sendo que, com as próteses modelo Carpentier (modelo 2526) de perfil mais elevado, as lesões encontrads foram do tipo I (junção atrioventricular), ao passo que, com a prótese Labcór de perfil mais baixo, a lesão era do tipo II (parede livre de VE). Em nenhum dos nossos pacientes o aparecimento da rotura do VE foi precedido de massagem cardiaca, ou outro tipo de manipulação do coração. Hematomas extensos, infiltrando o miocárdio em torno das lesōes, foram um achado constante.

\section{COMENTÁRIOS}

A resseção excessiva do músculo papilar, apontada, primeiramente, por ROBERTS \& MORROW ${ }^{24}$ e enfatizada por TREASURE et alli ${ }^{27}$ como um dos fatores de rotura do VE após substituição da valva mitral, pode ser afastada, em nossos pacientes, 3 dos quais foram imediatamente reoperados, enquanto que o quarto foi reexplorado no CTI. Em todos eles, a base do músculo papilar posterior apresentadava-se conservada.

Em nossos pacientes, uma infiltração sangüínea importante foi observada no sulco atrioventricular, na própria parede do VE e no músculo papilar, sempre envolvendo um ramo da artéria circunflexa, o que, aliás, dificultou muito as manobras para reparação das lesões. Parece-nos ser esta a razão pela qual muitos autores admitem que hematomas intramiocárdicos possam ser formados por tração excessiva no músculo papilar, ou no anel, levando à rotura (Figura 2).

ROBERTS \& MORROW ${ }^{24}$ atribuiram grande importância à presença de calcificação do anel valvar, particularmente na área da cúspide posterior, o que teria contribuído para uma super-exérese da valva mitral, comprometendo a parede do VE. Em nossos pacientes, cujos anéis valvares eram pouco fibrosos, havia cálcio em pequena quantidade em 3 deles, mas em nenhum encontramos calcificação no anel invadindo a parede do VE,

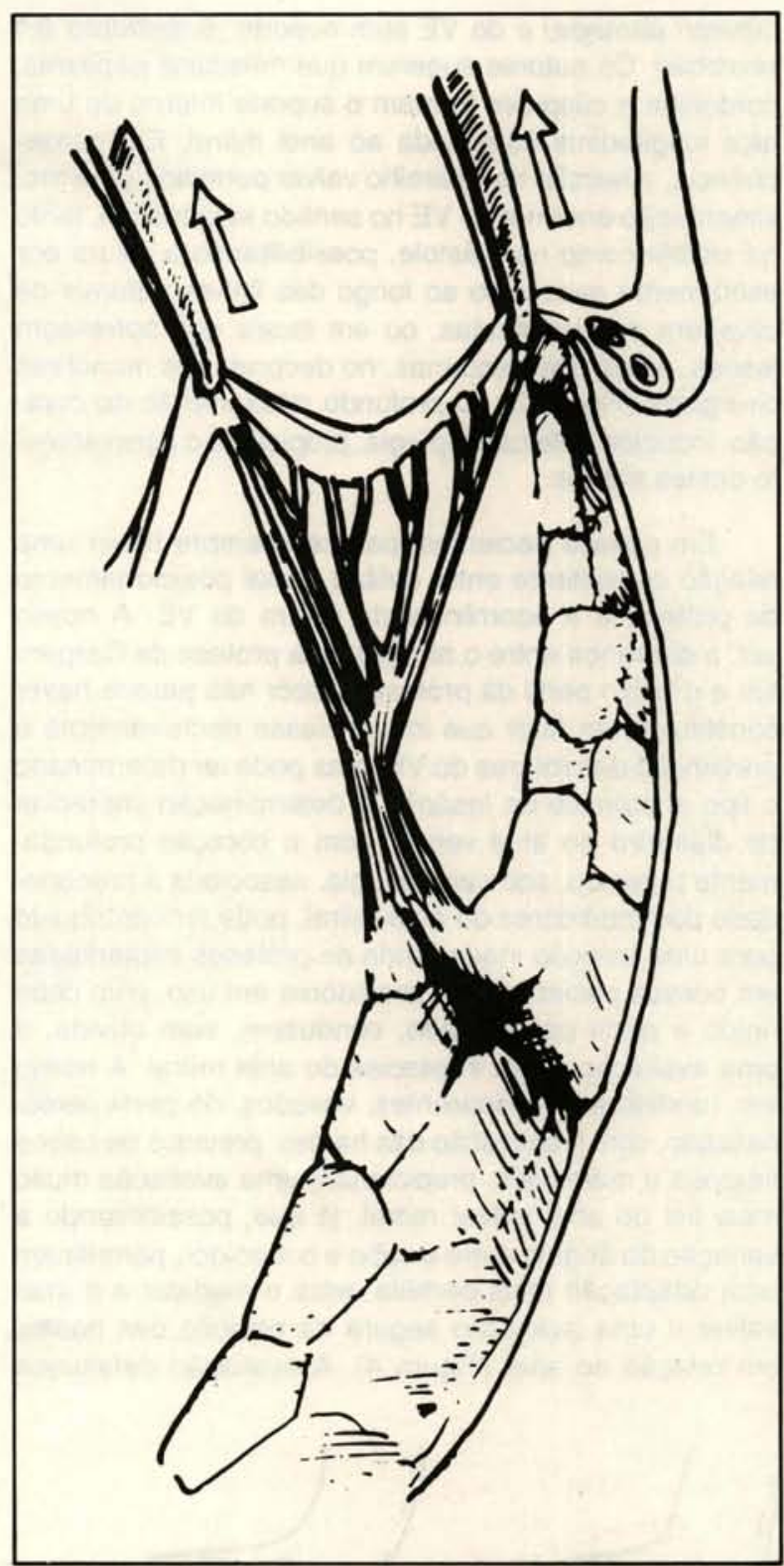

Fig. 2 - A traçăo excessiva do músculo ou do anel valvar, nas manobras do exérese da valva mitral, poderia conduzir à formaçăo de hematomas intramiocárdicos, que levariam à rotura da parede ventricular.

que pudesse justificar a ocorrência de uma rotura acidental da parede ventricular, nas manobras da exérese da valva.

Em 1964, LILLEHEI et alii ${ }^{19}$ propunham a conservaçăo do aparelho valvar mitral (cúspide, cordoalha $\mathbf{e}$ anel atrioventricular) em pacientes submetidos a substituição da valva mitral, com a finalidade de preservar a função do VE. Em 1977 e 1980, COBBS Jr. et alii 7 . ${ }^{8}$, baseados nos trabalhos de ARMOUR \& RANDAL ${ }^{2}$ e analisando os achados anatomopatológicos de 7 pacientes, introduziram o conceito de lesão de estiramento 
CHRISTO, M. C.; SOUZA, L. S. S.; STORTINI, M. J.; TEIXEIRA, S. L. C.; CRUZ JÚNIOR, O. - Rotura ventricular após substituiçấo da valva mitral. Rev. Bras. Cir. Cardiovasc., 4(1): 75-83, 1989.

(stretch damage) e do VE sem suporte (untethered left ventricle). Os autores sugerem que músculos papilares, cordoalha e cúspides formam o suporte interno de uma alça longitudinal conectada ao anel mitral. Em conseqüência, a secção do aparelho valvar permitiria uma movimentação anormal do VE no sentido longitudinal, tanto na sístole como na diástole, possibilitando a rotura por estiramento excessivo ao longo das linhas naturais de clivagem das trabéculas, ou em locais que sofressem lesões, ainda que pequenas, no decorrer das manobras cirúrgicas (Figura 3 ). O profundo relaxamento do coração, induzido pela cardioplegia, propiciaria o agravamento destes efeitos.

Em nossos pacientes, pareceu sempre haver uma relação consistente entre calibre e mal posicionamento da prótese e a ocorrẽncia da rotura do VE. A nosso ver, a diferença entre o alto perfil da prótese de Carpentier e o baixo perfil da prótese Labcór não parece haver constituido um fator que influenciasse decisivamente a prevenção das roturas do VE, mas pode ter determinado 0 tipo anatômico da lesão ${ }^{16}$. A determinação imprecisa do diâmetro do anel valvar, com o coração profundamente relaxado, sob cardioplegia, associada à precariedade dos medidores do anel mitral, pode ter contribuido para uma seleção inadequada de próteses implantadas em nossos pacientes. Os medidores em uso, com cabo rígido e perfil padronizado, conduzem, sem dúvida, a uma avaliação muito imprecisa do anel mitral. A nosso ver, medidores transparentes, vasados, de perfil personalizado, com localização das hastes, providos de cabos flexíveis e maleáveis, propiciariam uma avaliação muito mais fiel do anel valvar mitral, já que, possibilitando a variação do ângulo entre o cabo e o medidor, permitiriam uma adaptação mais perfeita entre o medidor e 0 anel valvar e uma avaliação segura da posição das hastes em relação ao anel (Figura 4). A avaliação defeituosa

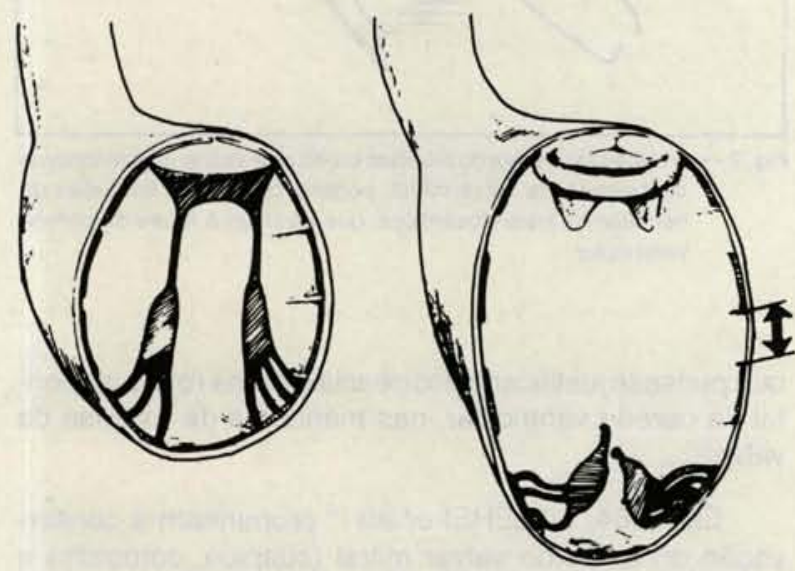

Fig. 3 - Reproduçăo esquemática da teoria do "ventriculo sem suporte" COBBS Jr. of ali $^{\mathbf{6}}$ : a exérese da valva mitral possibilitaria movimentaçăo anormal, no sentido longitudinal do VE, tanto na sístole, como na diástole, levando à rotura por estiramento excessivo, ao longo das linhas naturais de clivagem das trabé. culas, ou em locais que sofressem lesōes, ainda que pequenas. no decorrer das manobras cirúrgicas.

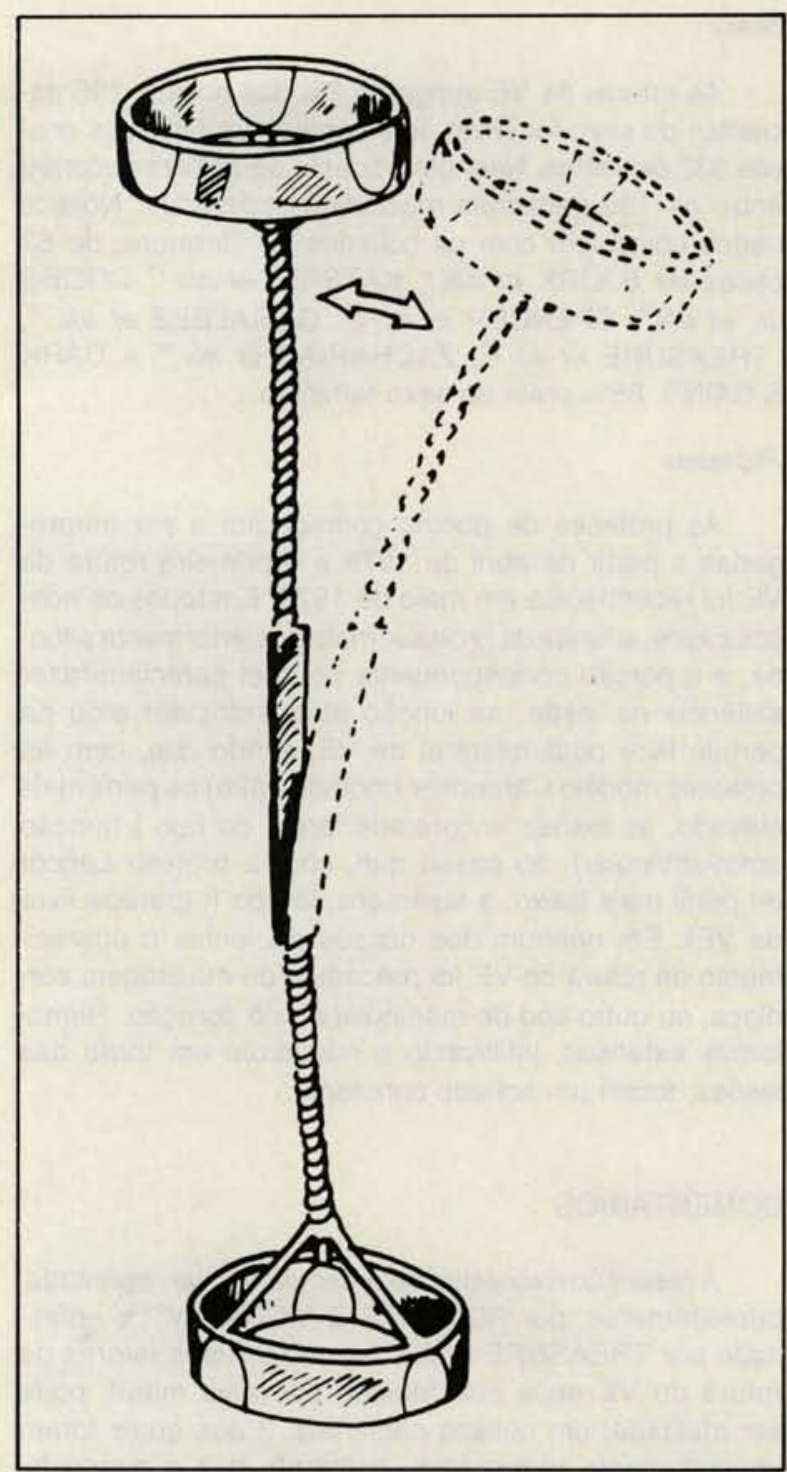

Fig. 4-A avaliaçâo imprecisa do anel mitral conduziria à escolha de próteses mal dimensionadas: medidores transparentes, vasados, de perfil personalizado, com localizaçăo das hastes, providos de cabos flexiveis e maleáveis propiciariam uma avaliaçāo muito mais fiel do anel mitral.

dos anéis valvares parece haver contribuido, em nossos pacientes, para a escolha de próteses mal calibradas e, em conseqüênia, viciosamente posicionadas. As próteses de calibre 31 e 33 foram usadas em $34 \%$ dos nossos pacientes, enquanto que as de menor calibre (29 e menos) constituíram $66 \%$ das próteses empregadas. Três das roturas ocorreram entre 111 pacientes com próteses 31 e 33 (2,7\% dos pacientes desse grupo).

Finalmente, parece perfeitamente cabivel comentar que, a nosso ver, a ocorrência das roturas de VE, após substituiçāo da valva mitral, decorre de uma congregação de vários fatores, precipitada pela seleção inadequada da prótese, particularmente em pacientes idosos, do sexo feminino, com anéis pouco fibrosos e atingindo 
CHRISTO, M. C.; SOUZA, L. S. S.; STORTINI, M. J.; TEIXEIRA, S. L. C.; CRUZ JÚNIOR, O. - Rotura ventricular após substituição da valva mitral. Rev. Bras. Cir. Cardiovasc., 4(1): 75-83, 1989.

uma região cuja irrigação parece ser basicamente deficiente, já que a ocorrência de lesōes isquêmicas graves atingindo o músculo papilar posterior é de 6 a 12 vezes mais freqüente do que lesōes isquêmicas semelhantes atingindo o músculo papilar anterior ${ }^{22}$. Coincidentemente, as roturas isquêmicas de VE são encontradas mais freqüentemente nas mulheres do que nos homens ${ }^{14},{ }^{18}$, 25 .

Concluindo, parece muito provável que uma prótese mal selecionada e viciosamente posicionada, forçando o anel mitral, em um "miocárdio sem suporte"7, 8, resultaria em isquemia local, criando área de acinesia em parede correspondente do VE. Em conseqüência, imediatamente após o retorno à atividade cardiaca, a porção imobilizada da parede começaria, a cada sístole, a se projetar para fora, em sentido contrário ao da sístole do miocárdio normal ${ }^{5,25}$, causando "lesōes miocárdicas por estiramento" $5,7,8$, associadas a roturas vasculares intramiocárdicas, com formação de hematoma progres-

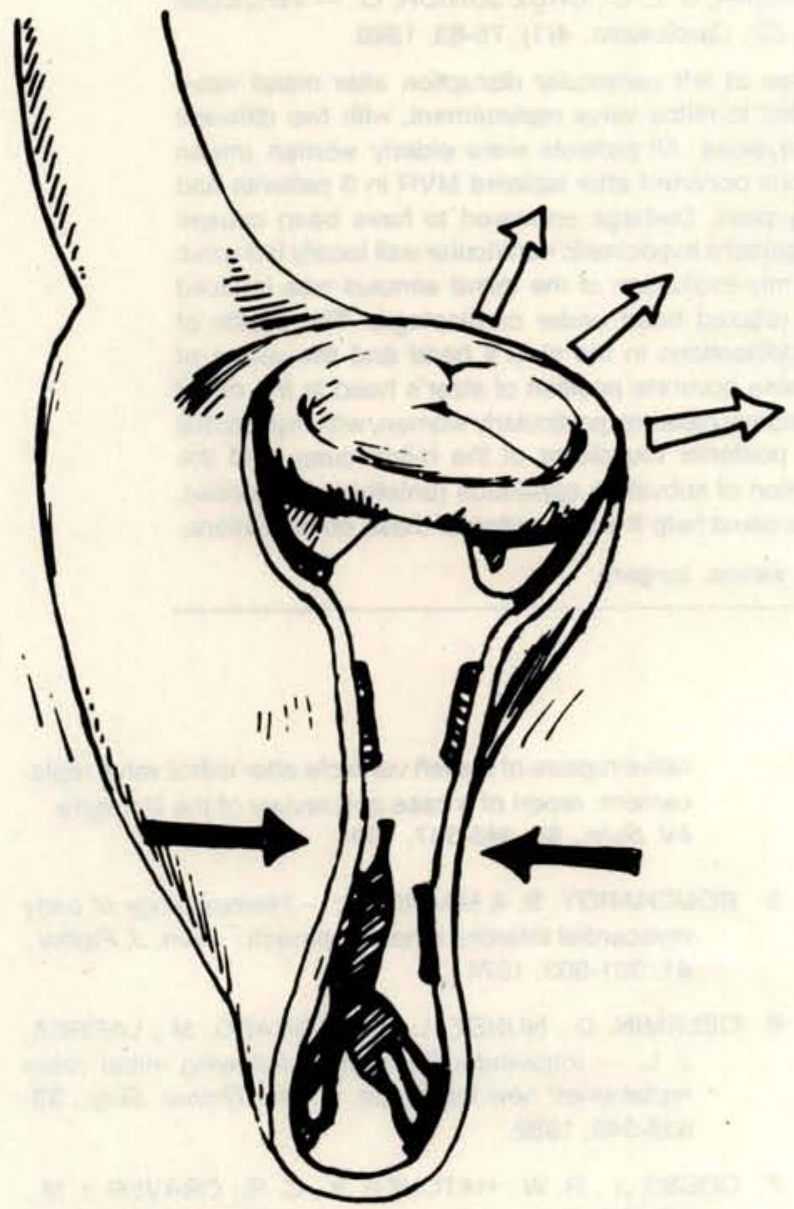

Fig. 5 - Uma prótese viciosamente implantada, forçando o anel mitral, provocando isquemia regional criaria uma situaçāo de acinesia localizada em parede correspondente do VE. Em conseqüência, a cada sistole, a porção imobilizada da parede ventricular se projetaria em sentido contrário ao da sístole do miocárdio normal, levando á rotura da parede ventricular. sivo invadindo a parede do $\mathrm{VE}^{7,8}$, resultando em rotura imediata da parede ventricular ou, tardiamente, em disfunçāo ventricular ou, ainda, na formaçāo de aneurismas locais ${ }^{8,16}$ (Figura 5). Condiçōes anatômicas e/ou patológicas pré-existentes em parede do VE determinariam o grau e a evolução da lesão ventricular.

Várias técnicas para reparação das roturas transversais do $V E$, envolvendo ou não a retirada da prótese, têm sido descritas ${ }^{6,13}$. Não é nosso propósito comentá-las, neste trabalho. Em nossa experiência, 3 roturas do tipo I, detectadas ainda no periódo pré-operatório (à saída de circulação extracorpórea) foram reparadas. Nos 3 csos, a prótese foi removida e a lesão, corrigida com o auxílio de um retalho de Teflon ou pericárdio bovino. Nos 3 casos, à reexploração, verificamos que as lesōes correspondentes eram, na face interna do coração, bem mais extensas do que pareciam quando examinadas por sua face externa. Nos 3 casos operados, a preservação das artérias circunflexas e/ou de um dos ramos envolvidos na lesão constituiu, sempre, uma dificuldade muito importante na parede lesada (Figura 6).

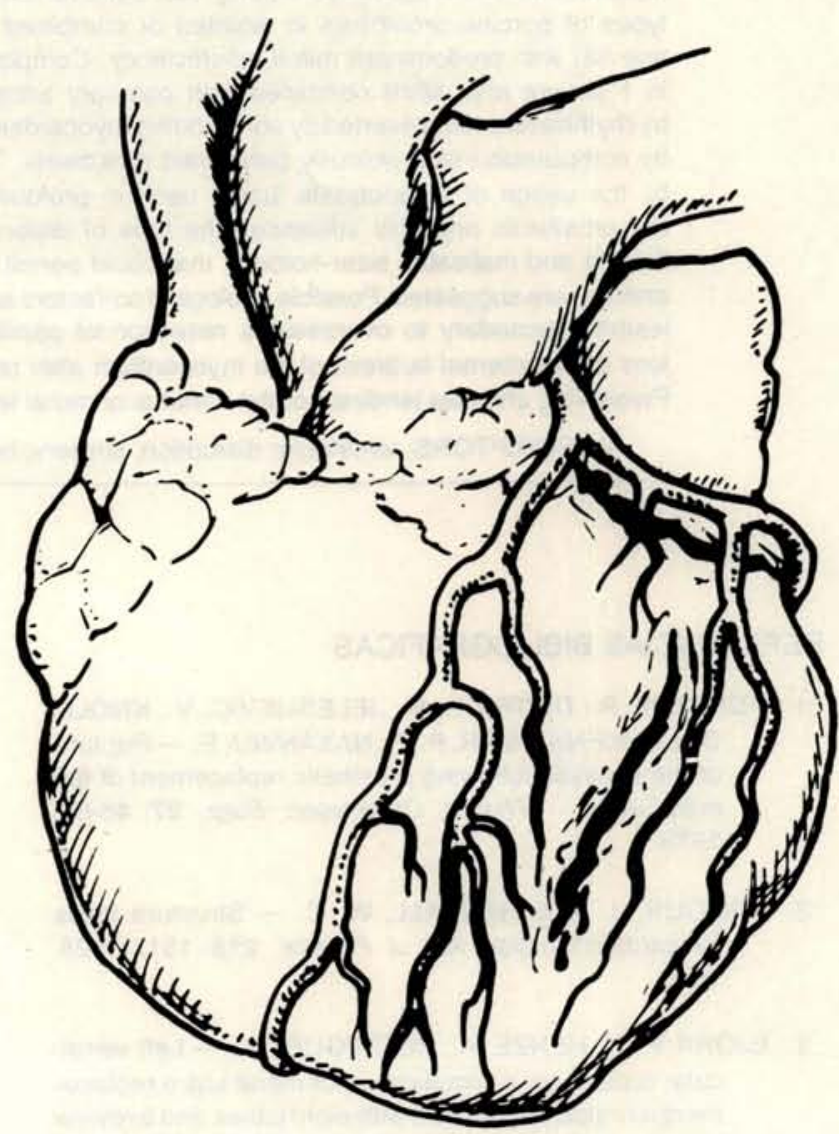

Fig. 6 - Nos 3 casos em que as lesóes do VE foram corrigidas, a preservaçăo das artérias circunflexas e/ou de seus ramos, envolvidas na lesão, constituiu, sempre, uma dificuldade muito importante. 
CHRISTO, M. C.; SOUZA, L. S. S.; STORTINI, M. J.; TEIXEIRA, S. L. C.; CRUZ JÚNIOR, O. - Rotura ventricular após substituição da valva mitral. Rev. Bras. Cir. Cardiovasc., 4(1): 75-83, 1989.

Mas, na realidade, os óbitos, nos 3 pacientes, ocorreram por baixo débito cardíaco conseqüentemente ao clampeamento prolongado da aorta, exigido para o tratamento da lesōes. A nosso ver, o emprego do balão intraaórtico teria aumentado as changes de recuperação desses pacientes*.

A preservaçāo do aparelho mitral no tratamento da valvopatia mitral seria muito desejável. Para tanto, o primeiro passo seria, sempre que possível, substituir as exéreses valvares por plastias. Entretanto, quando se

- Entretanto, este recurso nảo tem estado à disposiçăo dos cirurgiōes na maioria dos Serviços brasileiros, por não constituir exigència das entidades envolvidas nos Seguros de Saúde, ou do SIC-CV. fizesse necessária a substituição mitral, além de uma avaliação cuidadosa do calibre da prótese, que fosse sistematicamente preservado o aparelho mitral, se não completamente, pelo menos a cúspide posterior. Desde LILLEHEI et alii ${ }^{19}$, técnicas visando à preservação da cúspide mitral posterior têm sido propostas ${ }^{11,20}$. Não cabe, aqui, em estudo crítico desse material. Entretanto, cabe lembrar que a maioria dos cirurgiōes considera que a conservação de um aparelho valvar mitral sob uma prótese representaria, potencialmente, um foco de tromboembolismo e, além disso, seria problemática em presença de calcificação. Por outro lado, cumpre assinalar que, no excelente trabalho de SPENCER et alii ${ }^{26}$ no último de sua série de 14 pacientes com rotura do VE, a cúspide posterior havia sido conservada.

RBCCV 44205-79

CHRISTO, M. C.; SOUZA, L. S. S.; STORTINI, M. J.; TEIXEIRA, S. L. C.; CRUUZ JÚNIOR, O. - Ventricular disruption after mitral valve replacement. Rev. Bras. Cir. Cardiovasc., 4(1): 75-83, 1989.

ABSTRACT: Between 1979 and 1988 four fatal cases of left ventricular disruption after mitral valve replacement were registered among 332 patients submitted to mitral valve replacement, with two different types of porcine prosthesis in isolated or combined operations. All patients were elderly women (mean age 58) with predominant mitral insufficiency. Complications occurred after isolated MVR in 3 patients and in 1 patient after MVR combined with coronary artery by-pass. Damage appeared to have been caused by rhythmical pulling exerted by sorrounding myocardium against a hypocinetic ventricular wall locally ischemic by compression of a viciously positioned prosthesis. The mis-evaluation of the mitral annulus was induced by the usage of innadequate sizers used in profoundly relaxed heart under cardioplegia. The profile of the prosthesis probably influenced the type of lesion. Modifications in the sizer's head and the usage of flexible and malleable sizer-holders, that could permit a more accurate position of sizer's head in the mitral annulus are suggested. Possible etiological co-factors are: elderly patients, particularly women, with myocardial lesions secondary to overzealous resection of papillary posterior muscle or of the mitral cusp, and the loss of the internal buttress of the myocardium after resection of subvalvar apparatus (untethered ventricle). Preserving chordae tendinae of the annulus of mural leaflet could help the prevention of these complications.

DESCRIPTORS: ventricular disruption, surgery; heart valves, surgery.

\section{REFERÊNCIAS BIBLIOGRÁFICAS}

1 ACHATZY, R.; DITTRICH, H.; JELESIJEVIC, V.; KNOLL, D.; LUNKENHEIMER, P. P.; NAYANWU, E. - Rupture of the ventricle following prosthetic replacement of the mitral valve. Thorac. Cardiovasc. Surg., 27: 48-50, 1979.

2 ARMOUR, J. A. \& RANDALL, W. C. - Structure basis for cardiac function. Am. J. Physiol., 218: 1517-1523, 1970.

3 BJÖRK V. O.; HENZE, A.; RODRIGUEZ, L. - Left ventricular rupture as a complication of mitral valve replacement: surgical experience with eight cases and a review of literature. J. Thorac. Cardiovasc., Surg., 73: 14-21, 1977.

4 BORTOLOTTI, U.; LIVI, U.; MAZZUCCO, A.; GALLUCI, V.; CÉVESE, P. G. - Successful treatment of intraope- rative rupture of the left ventricle after mitral valve replacement: report of a case and review of the literature. Int. Surg., 66: 345-347, 1981.

5 BOUCHARDY, B. \& MAJNO, G. - Histopatology of early myocardial infarcts: a new approach. Am. J. Pathol., 41: 301-303, 1974.

6 CELEMIN, D.; NUNEZ, L.; GIL-AGUADO, M.; LARREA, J. L. - Intraventricular rupture following mitral valve - replacemet: new technique. Ann. Thorac. Surg., 33: 638-540, 1982.

7 COBBS Jr., R. W.; HATCHER Jr., C. R.; CRAVER, I. M.; JONES, E. L. - Disruption after mitral valve replacement. Circulation, 56(Supl. 3): 26, 1977.

8 COBBS Jr., R. W.; HATCHER Jr., C. R.; CRAVER, I. M.; JONES, E. L.; SEWELL, C. W. - Transverse mid-ventricular disruption after mitral valve replacement. $\mathrm{Am}$. Heart. J., 99: 33-50, 1980. 
$9 \mathrm{CHI}$, S.; BESGAVE, R.; GONZALEZ-LAVIN, I. - Left ventricular wall rupture after mitral valve replacement: report of successful repair in 2 patients. Ann. Thorac. Surg., 22: 380-382. 1976.

10 DARK, T. E. \& BAIN, W. H. - Rupture of posterior wall of the left ventricle after mitral valve replcement. Thorax, 39: 905-911, 1984.

11 DAVID, T. E. - Mitral valve replacement with preservation of chordae tendinae: rationale technical considerations. Ann. Thorac. Surg., 41: 680-682, 1986.

12 DEVINENI, R. \& McKENZIE, F. N. - Type I left ventricular rupture after mitral valve replacement. $J$. Thorac. Cardiovasc. Surg., 86: 742-745, 1983.

13 ENGLEMAN, R. M.; ROUSOU, J. H.; WITtEnBERG, S. A. - New technique for repair of posterior left ventricular rupture. J. Thorac. Cardiovasc. Surg., 77: 757-759, 1979.

14 FARAMARZ, N.; MAZA, L. M.; ROBINS, S. L. - Cardiac rupture during myocardial infarction: a review of 44 cases. Circulation, 55: 1231-1239, 1972.

15 gosAlbeZ, F.; LINERA, F. A.; COFIÑO, J. L.; NAYA, J. L.; RODRIGUEZ, J.; ORTUÑA, A. - Isolated mitral valve replacement and ventricular rupture: presentation of 6 patients. Ann. Thorac. Surg., 31: 105-110, 1981.

16 JONES, M.; EIDBO, E. E.; RODRIGUEZ, E. R.; FERRANS, V. J.; CLARK, R. E. - Ventricular aneurysms and other lesions produced by the struts of the bioprosthetic valves implanted in ship. J. Thorac. Cardiovasc. Surg., 95: $729-733,1988$.

17 KATSBIE, E.; GOLDING, L. R.; TUBBS, R. R.; LOOP, F. D. - Posterior mid-ventricular rupture after mitral valve replacement. Ann. Thorac. Surg., 27: 130-132, 1979.

18 LEWIS, A. J.; BURCEHLL, H. B.; TITUS. S. L. - Clinical and pathologic features of post infarction cardiac rupture. Am. J. Cardiol., 23: 43-53, 1969.

19 LILLEHEI, W.; LEVY, M. J.; BONNABEZU Jr., R. C. Mitral valve replacement with preservation of papilary muscles and chordae tendinae. J. Thorac. Cardio. vasc. Surg., 47: 532-543, 1964.

20 MILLER Jr., D. W.; JOHNSON, D. D.; IVEY, T. D. - Does preservation of the posterior chordae tendinae enhance survival during mitral valve replacement? Ann. Thorac. Surg., 28: 22-27, 1979.

21 MacVAUGH III, H.; JOYER, C. R.; JOHNSON, J. - Unusual complications during mitral valve replacement in the presence of calcification of annulus. Ann. Thorac. Surg., 11: 336-342, 1971.

22 MORROW, A. G.; COHEN, L. S.; ROBERTS, W. C.; BRAUNWALD, N. S. - Severe mitral regurgitation following acute myocardial infarction and rupture of pappilary muscle. Circulation, 37(Supl. 2): 124-132, 1968.
23 ROBERTS, W. C.; ISNER, J. T. VIRMANI, R. - Left ventricular incision midway between the mitral annulus and the stumps of the papillary muscle during mitral valve excision with or without rupture or aneurysmal formation: analysis of 10 necropsy patients. Am. Heart J., 6: 1278-1287, 1982.

24 ROBERTS, W. C.\& MORROW, A. G. - Causes of early postoperative death following cardiac valve replacement: clinico-pathologic correlations in 64 patients studied at necropsy. J. Thorac. Cardiovasc. Surg., 54: 422-437, 1967.

25 SIGLER, L. H. - Rupture of the heart in myocardial infarction: clinical and pathologic observations. Am. J. Cardiol., 5: 14-23, 1960.

SPENCER, F. C.; GALLAWAY, A. C.; COLVIN, S. B. A clinical evaluation of the hypothesis that rupture of the left ventricle following mitral valve replacement can be prevented by preservation of the chordae of the mural leaflet. Ann. Surg., 202: 673-680, 1985.

27 TREASURE, R. L.; RAINER, W. G.; STEVERY, R. E.; SADLER, T. R. - Intraoperative left ventricular rupture associated with mitral valve replacement. Chest, 66 : 511-514, 1974

28 WOLPOWITZ, A.; BARNARD, M. S.; SANCHEZ, H. F.; BARNARD, C. N. - Intraoperative posterior left ventricular rupture associated with mitral replacement. Ann. Thorac. Surg., 25: 551-554, 1978.

29 ZACHARIAS, A.; GROVES, L. K.; CHEANVECHAI C.; LOOP, F. D.; EFFLER, D. B. - Rupture of the posterior wall of the left ventricle after mitral valve replacement. J. Thorac. Cardiovasc. Surg., 69: 259-263, 1975.

\section{Discussão}

\section{DR. EDUARDO REGIS JUCÁ Fortaleza, CE}

Fico honrado com a oportunidade que me conferem de comentar o excelente trabalho do Dr. Campos Christo e associados, sobre tão importante tema. Nos nossos dias, poucos gostam de comentar sobre complicaçōes, morbidade e resultados adversos, esquecendo que analisando-os é que mais aprendemos. No nosso grupo, é rotina usar uma prótese mitral, menor do que a inicialmente estimada, não somente devido ao anel, mas também com receio de que as hastes da prótese batam na parede do ventrículo. Nós tivemos a oportunidade de reoperar 2 pacietes com próteses de pericárdio bovino, inseridas aqui em São Paulo, e a grande dificuldade não foi o anel; foi, justamente, de isolar as hastes do endocárdio da parede do ventrículo; o paciente havia recebido prótese do tamanho 31, portanto eu concordo plenamente com o Dr. Campos Christo, quando ele enfatiza a necessidade de colocar válvulas de diâmetro me- 
CHRISTO, M. C.; SOUZA, L. S. S.; STORTINI, M. J.; TEIXEIRA, S. L. C.; CRUZ JÚNIOR, O. - Rotura ventricular após substituicăo da valva mitral. Rev. Bras. Cir. Cardiovasc., 4(1): 75-83, 1989.

nor, o Dr. Robert, em trabalhos, enfatiza dados de necropsia que a causa mais perigosa é uma prótese mitral grande. No seu trabalho, o Dr. Campos Christo tem uma incidência baixa, se nós levarmos em consideraçāo que 2 dos pacientes tinham idade acima de 70 anos e um deles foi associado a ponte de safena. Na casa de Saúde São Raimundo, nós tivemos 1 paciente com rotura do ventrículo esquerdo tipo I; em 400 casos, mais ou menos, tivemos 3 casos idênticos; às vezes, pode ser um pouco maior que isso, porque alguns pacientes não vāo para necropsia: a incidência do Dr. Campos Christo é, realmente, a da literatura. O trabalho do Dr. A. Zacharias, um brasileiro que trabalha na Cleveland Clinic, dá, também, esse alto número; no trabalho do Dr. Svenson, a que o Dr. Campos Christo se referiu, estāo citados 2 casos, em 14, e entăo propós que, ao final da cirurgia da troca da valva mitral, levante-se a ponta do coração, para se ver se há algum hematoma, o que propiciou um editorial do Dr. Björk condenando isto; um movimento de báscula, que o Dr. Campos Christo citou, no caso dele, com ponte de safena associada, eu perguntaria se ele fez primeiro a ponte, ou primeiro a troca, porque a nossa conduta é tirar a valva, fazer a ponte e, depois, inserir a valva no lugar. Concluindo, congratulo-me com o Dr. Campos Christo, pela oportunidade do trabalho, pela excelência do mesmo e pergunto se, depois dessa análise retrospectiva, ele tem algum episódio desse tipo; em segundo lugar, se ele condena tanto a cardioplegia, eu pergunto se mudou propriamente soltando a aorta e estimulando melhor o anel; em terceiro lugar, pergunto se, no seu trabalho, só usou próteses biológicas e se, em alguma oportunidade, usa prótese mecânica. Era o que eu tinha a dizer. Muito obrigado.

\section{DR. RANDAS VILELA BATISTA Curitiba, $P R$}

Agradeço à Comissão Organizadora, pelo convite e pelo envio prévio do manuscrito do trabalho aqui apresentado e que tenho a honra de comentar. Nossa experiência tem sido relativamente igual, isto é, nos últimos 5 anos, nós operamos 386 pacientes e tivemos 3 roturas de ventrículo esquerdo, sendo 2 roturas do tipo 1 e 1 rotura do tipo II. Discordo de algumas exposições do Dr. Campos Christo, no seguinte sentido: quanto ao anel calcificado, isto é muito importante; ontem mesmo, estávamos dissecando uma valva que estava massivamente calcificada e, ao sair a valva, nós notamos o músculo do ventrículo esquerdo e o músculo do átrio e, no meio, a gordura; então, imediatamente, fez-se uma reaproximação do ventrículo ao átrio e náo houve problemas, graças à experiência passada, porque, no primeiro caso em que tivemos complicação, nós notamos uma hemorragia na área da circunflexa e não demos valor, e o paciente veio a óbito na UTI; o segundo caso, quando nós vimos a mesma imagem de hematoma na região da circunflexa, voltamos à circulação extracorpórea e reaproximamos o ventrículo do átrio; assim, em pacien- tes com anel muito calcificado, a tendência do cirurgiăo é ressecar quanto mais cálcio possível $e$, dependendo do caso, se todo o cálcio foi ressecado, vai-se terminar no pericárdio. Quanto á idade, acho que é importante, nas não a do paciente, mas a do cirurgiăo; um cirurgiăo mais experiente tem menos possibilidade de ter esse problema; quanto ao sexo, a literatura diz que, realmente, a incidência é maior no sexo feminino; eu, no entanto, nāo acredito que o sexo tenha importância; é apenas mera coincidência; acontece que, nas mulheres, os coraçōes são menores; então, se formos avaliar superfície corporal com massa cardiaca, vai-se notar que as mulheres têm menor massa cardíaca, o que predispōe ao problema. E, finalizando, eu gostaria de perguntar ao Dr. Campos Christo com referência ao paciente que teve rotura tipo II. Nós tivemos um paciente no qual eu achei que havia ressecado muito o músculo papilar e eu dizia ao anestesista: o paciente é hipertenso, por favor não deixe a pressão passar de 100; dito e feito, ao fechar o esterno, uma hemorragia profusa veio do ventrículo, olhei a pressão, estava em 200 e, simplesmente, o ventrículo rachou em dois. Essas coisas acontecem, sempre, no início da experiência do cirurgiâo. Cumprimento o Dr. Campos Christo e colaboradores pelo excelente trabalho aqui apresentado. Obrigado.

\section{DR. CAMPOS CHRISTO (Encerrando)}

Agradeço aos Drs. Jucá e Randas pelos comentários. Na seqüência, nós colocamos, primeiramente, a ponte, para, depois, substituirmos a valva. Quanto ao problema do acidente, acho que tivemos somente 1 , há 1 ano e não é questão da experiência do cirurgião, ou não; é questão de uma série de fatores: eram pacientes idosos e acho que a questão idade tem uma importância fundamental; é um dado do qual não podemos abrir mão; realmente, quando vamos operar um doente idoso, se for mulher, entāo, é um dado fundamental; quanto a isto, acho que não podemos fazer concessão nenhuma; agora, quanto ao problema, por exemplo, do sexo, na realidade atinge mais o sexo feminino, não tenho dúvida; revi toda a literatura e os dados aí apresentados comprovam que as roturas expontâneas do ventrículo esquerdo após isquemias agudas depois de infarto, são mais freqüentes na mulher do que no homem; realmente, os coraçōes se rompem mais expontaneamente nas mulheres porque são corações de paredes mais delgadas, mais finas. O problema da ressecção do anel calcificado, que o Dr. Randas enfatizou, quero chamar a atenção para o fato de que os nossos pacientes não tinham calcificação; além do mais, esses acidentes não ocorrem só nas mãos de um cirurgiăo, mas nas de um grupo e essa freqüência é constante em todas as revisōes da literatura; nós vimos que, constantemente, ocorrem de $0,5 \%$ a $2,0 \%$. O Dr. Randas referiu-se a uma maneira muito simples de se corrigir a lesão e isto é uma outra coisa que eu gostaria de chamar a atenção; 
CHRISTO, M. C.; SOUZA, L. S. S.; STURTINI, M. J.; TEIXEIRA, S. L. C.; CRUZ JÚNIOR, O. - Rotura ventricular após substituiçăo da valva mitral. Rev. Bras. Cir. Cardiovasc., 4(1): 75-83, 1989.

a correção extremamente difícil é uma das tarefas mais dificultosas que tem o cirurgião, tanto que não existem técnicas, ora retira-se a prótese por dentro, ora tenta-se corrigir por fora; eu também concordo em que é, realmente, um acidente gravíssimo e de correção extremamente difícil. Outro fato para o qual desejo chamar a atenção é que as comissões que fiscalizam o funcionamento dos Serviços deveriam exigir, em todos eles, a presença do balão intra-aórtico; deveriam colocar, como condiçāo sine qua non para o funcionamento de um Serviço de Cirurgia Cardiovascular, a existência de um balão intra-aórtico. Era o que eu tinha a dizer. Muito obrigado. 